\title{
Stochastic Hybrid Combining Design for Quantized Massive MIMO Systems
}

\author{
Yalin Wang, Xihan Chen, Yunlong Cai, and Lajos Hanzo
}

\begin{abstract}
Both the power-dissipation and cost of massive multiple-input multiple-output (mMIMO) systems may be substantially reduced by using low-resolution analog-to-digital converters (LADCs) at the receivers. However, both the coarse quantization of LADCs and the inaccurate instantaneous channel state information (ICSI) degrade the performance of quantized mMIMO systems. To overcome these challenges, we propose a novel stochastic hybrid analog-digital combiner (SHC) scheme for adapting the hybrid combiner to the long-term statistics of the channel state information (SCSI). We seek to minimize the transmit power by jointly optimizing the SHC subject to average rate constraints. For the sake of solving the resultant nonconvex stochastic optimization problem, we develop a relaxed stochastic successive convex approximation (RSSCA) algorithm. Simulations are carried out to confirm the benefits of our proposed scheme over the benchmarkers.
\end{abstract}

\section{INTRODUCTION}

Massive multiple-input multiple-output (mMIMO) systems constitute promising techniques for next generation wireless communication [1]. The number of antennas is scaled up compared to traditional MIMO systems with the objective of improving both the energy efficiency (EE) and spectral efficiency (SE) of communications, albeit at the cost of increasing the power-dissipation of both the signal-processing and RF hardware. As a remedy, hybrid analog-digital combining aims for reducing the power by employing fewer radio frequency (RF) chains linked to a large number of antennas by an analog combiner [2]. Since the power-dissipation of analogto-digital converters (ADCs) increases exponentially with the number of quantization bits, low-resolution ADCs (LADCs) are desired [3]. Therefore, the seamless integration of hybrid combining and LADCs is of paramount importance.

Significant efforts have been made to study the feasibility of hybrid analog-digital combining aided quantized mMIMO systems relying on LADCs. Specifically, the authors of [3] developed hybrid combiners by minimizing the mean-squared error of received signals in the presence of multiuser interference. However, their analog combiner design does not satisfy the constant modulus constraint, hence its implementation is impractical. To circumvent this difficulty, the authors of [4] conceived a more practical hybrid combiner relying on a partially-

Copyright (c) 2015 IEEE. Personal use of this material is permitted. However, permission to use this material for any other purposes must be obtained from the IEEE by sending a request to pubs-permissions@ieee.org.

The work of Y. Cai was supported in part by the National Natural Science Foundation of China under Grants 61831004 and 61971376, the Zhejiang Provincial Natural Science Foundation for Distinguished Young Scholars under Grant LR19F010002, the National Key R\&D Program of China (No.2020YFB1805005), and the State Key Laboratory of Rail Traffic Control and Safety (Contract No. RCS2020K010), Beijing Jiaotong University.

L. Hanzo would like to acknowledge the financial support of the European Research Council's Advanced Fellow Grant QuantCom. (Correspondence authors: Xihan Chen; Yunlong Cai.)

Y. Wang and X. Chen are with the College of ISEE, Zhejiang University, Hangzhou 310027, China (e-mail: wang_yalin@zju.edu.cn; chenxihan@zju.edu.cn). Y. Cai is with the College of ISEE, Zhejiang University, Hangzhou 310027, China, and also with the State Key Laboratory of Rail Traffic Control and Safety, Beijing Jiaotong University, Beijing 100044, China (email: ylcai@zju.edu.cn). L. Hanzo is with the Department of ECS, University of Southampton, Southampton SO17 1BJ, U.K. (e-mail: 1h@ecs.soton.ac.uk). connected structure and rigorously derived the achievable rate considering the correlation of quantization errors. To further mitigate the effect of quantization errors, a two-stage analog combiner was developed in [5] for maximizing the mutual information between the quantized and the transmitted signals. Nevertheless, the aforementioned studies merely optimize the hybrid combiner in a separate manner, which inevitably leads to performance degradation. To mitigate this impediment, the authors of [6] harnessed fractional programming techniques for EE maximization by opting for the joint hybrid combiner design principle.

However, to the best of our knowledge, the studies in the literature mainly depend on the knowledge of the instantaneous channel state information (ICSI). In practice, acquiring ICSI is quite challenging in mMIMO systems. Owing to the limited coherence time associated with a high number of antennas, an excessive number of pilot symbols is necessitated for accurate channel estimation in the mMIMO regime. A further problem is the severe nonlinear distortion due to the coarse quantization by LADCs. Fortunately, the base station (BS) is capable of acquiring the slowly-varying statistical channel state information (SCSI) with the aid of its long-term feedback [7]. Therefore, it is more reasonable to consider a hybrid combining scheme purely relying on the knowledge of SCSI. Moreover, the pioneering contribution of [8] intimates the underlying EE vs SE trade-off. On the other hand, most of the existing studies such as [6] and [9] only place particular emphasis on improving the EE of quantized mMIMO systems. As such, how to design hybrid combining schemes based on the knowledge of SCSI for quantized MMIMO systems when the trade-off between the EE and SE is explicitly considered deserves further study.

To shed more light on these critical issues, we devise a novel stochastic hybrid combiner (SHC) scheme for quantized mMIMO systems and assume that only the SCSI is available at the BS. By invoking beamspace mMIMO techniques which steer the arriving signals having various angles of arrival to distinct array elements, we can significantly reduce the number of RF chains and conceive cost-efficient implementations. Our interest in this compact paper lies in striking a compelling throughput vs. power consumption trade-off, namely a SE vs. EE trade-off. To this end, we seek to minimize the transmit power by jointly optimizing the SHC scheme subject to average rate constraints. For efficiently solving this nonconvex stochastic constrained optimization problem, we propose a relaxed stochastic successive convex approximation (RSSCA) algorithm, which intrinsically amalgamates a binary relaxation technique with a stochastic successive convex approximation (SSCA) solution. The proposed SHC scheme only adapts the hybrid combiner to the long-term SCSI and offers compelling advantages over the existing schemes. Firstly, as a benefit of the channel hardening phenomenon of mMIMO systems [10], 
a fading channel behaves almost deterministically. Hence, considering the hybrid combiner relying on SCSI is more practical and efficient, yet without substantial performance degradation. Secondly, the hybrid combiner is only updated with the aid of the outdated CSI samples, hence mitigating the CSI signaling latency. Our simulation results validate that the proposed scheme outperforms the benchmarkers.

\section{System Model And Problem Formulation}

\section{A. Network Architecture and Frame Structure}

We consider a single-cell multiuser quantized mMIMO uplink system, where a BS supports $K$ users. Each user is equipped with a single antenna and the BS is equipped with $M>1$ antennas and $S \ll M$ RF chains. We focus on a fully-connected RF combining structure at the BS, where each RF chain is connected to all receive antennas using phase shifters [14] and LADCs are employed between the RF combiner and the digital combiner for converting the analog signals to discrete-amplitude signals. To enable the reliable retrieval of data symbols for each user, a low-complexity linear receive beamformer $\mathbf{w}_{k} \in \mathbb{C}^{S \times 1}$ is also employed at the BS. Specifically, the received signal vector is combined by a hybrid combiner using LADCs, as shown in Fig. 1.

Let $p_{k}$ be the transmit power of user $k, \mathbf{h}_{k} \in \mathbb{C}^{M \times 1}$ be the uplink channel spanning from user $k$ to the BS, $s_{k} \sim$ $\mathcal{C N}(0,1)$ be the transmitted data of user $k$, and $\mathbf{n} \in \mathbb{C}^{M \times 1}$ be the additive white Gaussian noise (AWGN) at the BS with distribution $\mathcal{C N}\left(0, \sigma^{2} \mathbf{I}_{M}\right)$. Assuming a narrowband channel, the signal received at the $\mathrm{BS}$ can be expressed as

$$
\mathbf{y}=\sum_{k=1}^{K} \sqrt{p_{k}} \mathbf{h}_{k} s_{k}+\mathbf{n}=\mathbf{H P}^{\frac{1}{2}} \mathbf{s}+\mathbf{n},
$$

where $\mathbf{P}=\operatorname{diag}\left(p_{1}, \cdots, p_{k}\right), \mathbf{H}=\left[\mathbf{h}_{1}, \cdots, \mathbf{h}_{K}\right] \in \mathbb{C}^{M \times K}$, and $\mathbf{s}=\left[s_{1}, \cdots, s_{k}\right]^{T}$.

The received signal $\mathbf{y}$ is first combined by the RF combiner $\mathbf{U} \in \mathbb{C}^{M \times S}$, which is implemented based on a discrete Fourier transform (DFT) codebook D. Consequently, such a DFT codebook-based RF combiner can be formulated as $\mathbf{U}=\mathbf{D C}$, where $\mathbf{D}=\left[\mathbf{d}_{1}, \cdots, \mathbf{d}_{N}\right] \in \mathbb{C}^{M \times N}$ denotes the codebook of size $N$ and $\mathbf{C} \in \mathbb{C}^{N \times S}$ denotes the selection matrix with element $c_{i j} \in\{0,1\}$ for selecting codewords. It can be readily implemented utilizing a static phase shifting network in the RF domain along with an RF switch. Therefore, the output of the RF combiner can be expressed as $\tilde{\mathbf{y}}=\mathbf{U}^{H}\left(\mathbf{H P}^{\frac{1}{2}} \mathbf{S}+\mathbf{n}\right)$. Each of the RF combining output $\tilde{\mathbf{y}}$ is connected to a LADC pair, which separately quantizes the imaginary and real part of the signal $\tilde{\mathbf{y}}$. Considering that each of the LADC has $q$ quantization bits, the quantized output under the additive quantization noise model (AQNM) [6] is expressed as

$$
\mathbf{y}_{q}=f(\tilde{\mathbf{y}})=\mathbf{Q}_{\gamma} \tilde{\mathbf{y}}+\mathbf{n}_{q}=\mathbf{Q}_{\gamma} \mathbf{U}^{H} \mathbf{H} \mathbf{P}^{\frac{1}{2}} \mathbf{S}+\mathbf{Q}_{\gamma} \mathbf{U}^{H} \mathbf{n}+\mathbf{n}_{q}, \text { (2) }
$$

where $f(\cdot)$ is the element-wise quantization function, $\mathbf{Q}_{\gamma}=$ $\operatorname{diag}(\gamma, \cdots, \gamma) \in \mathbb{C}^{S \times S}$ with quantization gain $\gamma=1-\rho$, and $\rho$ is a normalized quantization error. For $\rho \leq 5$, the typical values of $\rho$ are listed in [12], while for $\rho \geq 5$, they can be approximated by $\rho=\frac{\pi \sqrt{3}}{2} 2^{-2 q}$. The additive quantization noise $\mathbf{n}_{q}$ obeys the complex-valued Gaussian distribution with zero mean so that $\mathbf{n}_{q}$ and $\tilde{\mathbf{y}}$ are uncorrelated with each other. For a fixed channel realization $\mathbf{H}$, the covariance matrix of $\mathbf{n}_{q}$ is written as $\mathbf{R}_{q}=\mathbb{E}\left[\mathbf{n}_{q} \mathbf{n}_{q}^{H}\right]=\mathbf{Q}_{\gamma}(\mathbf{I}-$ $\left.\mathbf{Q}_{\gamma}\right) \operatorname{Diag}\left(\mathbf{U}^{H} \mathbf{H} \mathbf{P} \mathbf{H}^{H} \mathbf{U}+\sigma^{2} \mathbf{U}^{H} \mathbf{U}\right)$. Once the received signals are quantized, the baseband combiner $\mathbf{V} \in \mathbb{C}^{S \times S}$ at the $\mathrm{BS}$ is applied to handle the quantization noise introduced by the LADCs, additionally mitigating the multiuser interference. The corresponding output is given by

$$
\overline{\mathbf{y}}=\mathbf{V}^{H} \mathbf{y}_{q}=\mathbf{V}^{H} \mathbf{Q}_{\gamma} \mathbf{U}^{H} \mathbf{H} \mathbf{P}^{\frac{1}{2}} \mathbf{s}+\mathbf{V}^{H} \mathbf{Q}_{\gamma} \mathbf{U}^{H} \mathbf{n}+\mathbf{V}^{H} \mathbf{n}_{q}
$$

Finally, the retrieved data symbol of user $k$ after the lowcomplexity linear receive beamformer can be expressed as

$$
\hat{s_{k}}=\mathbf{w}_{k}^{H} \mathbf{V}^{H} \mathbf{Q}_{\gamma} \mathbf{U}^{H} \mathbf{H} \mathbf{P}^{\frac{1}{2}} \mathbf{s}+\mathbf{w}_{k}^{H} \mathbf{V}^{H} \mathbf{Q}_{\gamma} \mathbf{U}^{H} \mathbf{n}+\mathbf{w}_{k}^{H} \mathbf{V}^{H} \mathbf{n}_{q}
$$

By referring to the system model, the instantaneous rate of user $k$ can be explicitly expressed as $r_{k}(\mathbf{P}, \mathbf{C}, \mathbf{V}, \mathbf{W})=$ $\log \left(1+\operatorname{SINR}_{k}\right)$, where $\mathbf{W}=\left[\mathbf{w}_{1}, \cdots, \mathbf{w}_{K}\right] \in \mathbb{C}^{S \times K}$ is the composite receive beamforming vector and $\mathrm{SINR}_{k}$ denotes the received signal-to-interference-plus-noise ratio (SINR) of user $k$ defined in (5), shown at the bottom of this page. Hence, the average rate of user $k$ is given by $\bar{r}_{k}(\mathbf{P}, \mathbf{C}, \mathbf{V}, \mathbf{W})=$ $\mathbb{E}\left[r_{k}(\mathbf{P}, \mathbf{C}, \mathbf{V}, \mathbf{W})\right]$.

Remark 2.1: In contrast to the conventional hybrid combiner structure based on the knowledge of ICSI, both the RF combiner $\mathbf{U}$ and the baseband combiner $\mathbf{V}$ are only adapted to the SCSI in our proposed SHC scheme. By exploiting the channel hardening property of quantized mMIMO systems, the gain of adapting the power allocation based on the ICSI remains modest [11]. Hence, we adapt the power allocation strategy $\mathbf{P}$ to the long-term SCSI in consideration of the signaling overhead.

\section{B. Frame Structure}

In the proposed SHC scheme, we divide the time domain into several super-frames and each super-frame consists of $L_{f}$ frames. We further divide each frame into $L_{s}$ time slots, where the channel remains constant within each time slot. Thanks to the advanced compressive sensing based channel estimation methods, it is possible to achieve efficient uplink training with the aid of a limited number of RF chains [14]. Under this setup, we can obtain one (possibly outdated) channel sample $\mathbf{H}$ at each frame. To be more specific, each user sends dedicated uplink pilots to the BS, and the effective channel gains are subsequently estimated based on the received pilot signals. It is noteworthy that the variables $\mathbf{P}, \mathbf{C}, \mathbf{V}, \mathbf{W}$ are only updated once based on a single channel sample in each frame to achieve a mMIMO array gain at a reduced implementation cost.

C. Problem Formulation

We are interested in designing a hybrid combiner for the uplink of quantized mMIMO systems for minimizing

$$
\operatorname{SINR}_{k}=\frac{p_{k}\left|\mathbf{w}_{k}^{H} \mathbf{V}^{H} \mathbf{Q}_{\gamma} \mathbf{U}^{H} \mathbf{h}_{k}\right|^{2}}{\sum_{i \neq k} p_{i}\left|\mathbf{w}_{k}^{H} \mathbf{V}^{H} \mathbf{Q}_{\gamma} \mathbf{U}^{H} \mathbf{h}_{i}\right|^{2}+\sigma^{2}\left\|\mathbf{w}_{k}^{H} \mathbf{V}^{H} \mathbf{Q}_{\gamma} \mathbf{U}^{H}\right\|^{2}+\mathbf{w}_{k}^{H} \mathbf{V}^{H} \mathbf{R}_{q} \mathbf{V} \mathbf{w}_{k}}
$$




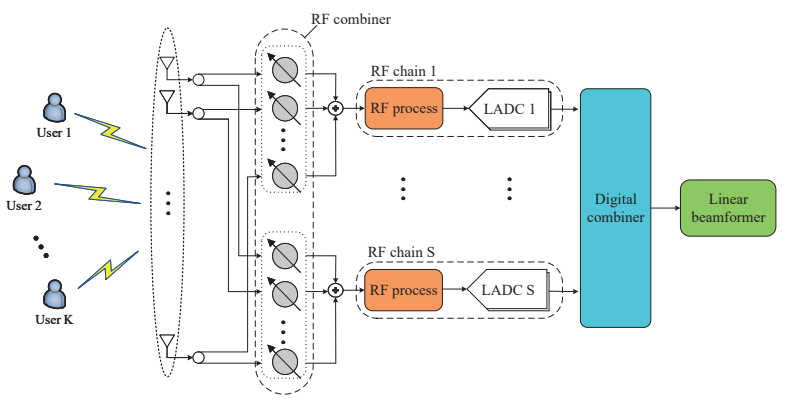

Fig. 1. An illustration of the SHC scheme in a multi-user mMIMO uplink system with LADCs.

the system's power-dissipation subject to the average rate requirement of each user. In practical implementations power control is of pivotal importance, especially in the uplink of quantized mMIMO system. Upon reducing the transmit power, the battery life of power constrained devices will be commensurately prolonged. In particular, the DFT-based RF combiner is adopted in the SHC for striking a performance vs. hardware cost trade-off, where the choice of codewords must strictly meet the following two criteria: 1) each RF chain is associated with a single codeword, 2) each codeword is assigned to no more than one RF chain. We consider the average rate as our QoS metric and denote the target rate requirement of user $k$ by $\gamma_{k}>0$. More formally, the problem can be formulated as

$$
\begin{aligned}
\min _{\mathbf{P}, \mathbf{C}, \mathbf{V}, \mathbf{W}} & \sum_{k=1}^{K} p_{k} \\
\text { s.t. } \quad & \bar{r}_{k}(\mathbf{P}, \mathbf{C}, \mathbf{V}, \mathbf{W}) \geq \gamma_{k}, \quad \forall k, \\
& p_{k} \leq P_{k}^{\max }, \quad \forall k, \\
& \sum_{i=1}^{N} c_{i j}=1, \quad \forall j, \\
& \sum_{j=1}^{S} c_{i j} \leq 1, \quad \forall i, \\
& c_{i j} \in\{0,1\}, \quad \forall i, j,
\end{aligned}
$$

where (6b) is the average rate requirement and (6c) is the transmit power constraint of each user. The constraints $(6 \mathrm{~d})$ and (6e) guarantee the realization of codewords selection criteria.

\section{Stochastic Hybrid Combining Scheme}

There are three major challenges in solving problem (6): i) the nonconvexity of the constraint functions, mainly due to the coupling variables in constraint (6b) and the discrete binary variable $c_{i j}$ in (6f); ii) the NP-hard property of the mixed integer nonlinear programming caused by (6f); and iii) the stochastic nature of constraint (6b). In the sequel, we develop an efficient algorithm to address this problem iteratively.

\section{A. Problem Transformation and Surrogate Function}

To tackle the difficulty arising from the discrete feasible region, we replace constraint (6f) that $c_{i j}$ be 0 or 1 with the relaxed constraint that it be in the interval $[0,1]$. To obtain an integer solution for the codeword selection indicator, we rely on the method of [14] to round each $\hat{c}_{i j}$ generated by the proposed algorithm to the nearest integer as follows.

$$
c_{i j}= \begin{cases}\left\lfloor\hat{c}_{i j}\right\rfloor=0, & \text { if } \hat{c}_{i j}-\left\lfloor\hat{c}_{i j}\right\rfloor \leq \varepsilon_{j}, \forall i, j, \\ \left\lceil\hat{c}_{i j}\right\rceil=1, & \text { otherwise, }\end{cases}
$$

where $0 \leq \varepsilon_{j} \leq 1$ is chosen using a simple bisection method, so that both the constraints (6d) and (6e) are met.

For convenience, we let $\mathbf{p}=\operatorname{diag}(\mathbf{P}), \mathbf{c}=\operatorname{vec}(\mathbf{C})$, $\mathbf{v}=\operatorname{vec}(\mathbf{V}), \mathbf{w}=\operatorname{vec}(\mathbf{W})$ and define variable $\mathbf{x} \triangleq$ $\left[\mathbf{p}^{T}, \mathbf{c}^{T}, \mathbf{v}^{T}, \mathbf{w}^{T}\right]^{T} \in \chi$, where $\chi$ is a convex constraint set defined in (8) as displayed at the bottom of this page. The key observation is that $\chi$ has a decoupled form: $\chi=\left\{\mathbf{x}: x_{t} \in\right.$ $\left.\chi_{t}, t=1, \ldots, n\right\}$, where $\chi_{t}$ is a convex region in $\mathbb{C}$. Then constraint (6b) can be rewritten as $f_{k}(\mathbf{x}) \triangleq \gamma_{k}-\bar{r}_{k}(\mathbf{x}) \leq 0$. It is challenging to accurately calculate the expectations in the constraint function $f_{k}(\mathbf{x})$. To handle such a stochastic constraint, we first construct the quadratic surrogate function $\hat{f}_{k}^{l}(\mathbf{x})$ of the constraint function $f_{k}(\mathbf{x})$, which efficiently handles the expectations and facilitates rapidly-converging algorithm design at a low complexity. $\hat{f}_{k}^{l}(\mathbf{x})$ can be recognized as a convex approximation of $f_{k}(\mathbf{x})$ at each iteration. Each channel sample corresponds to an iteration. Let $\mathrm{x}^{l}$ denote the variable used during the $l$-th channel sample. Specifically, $\hat{f}_{k}^{l}(\mathbf{x})$ is formulated as

$$
\hat{f}_{k}^{l}(\mathbf{x})=\gamma_{k}-\hat{r}_{k}^{l}+\Re\left[\left(\boldsymbol{\kappa}_{k}^{l}\right)^{H}\left(\mathbf{x}-\mathbf{x}^{l}\right)\right]+\tau_{k}\left\|\mathbf{x}-\mathbf{x}^{l}\right\|^{2},
$$

where $\tau_{k}$ is a positive constant so that it ensures the strong convexity of $\hat{f}_{k}^{l}(\mathbf{x})$. For given channel samples $\mathbf{H}^{i}, \hat{r}_{k}^{l}=$ $\sum_{i}^{l} r_{k}\left(\mathbf{x}^{l} ; \mathbf{H}^{i}\right) / l, \forall i=1, \ldots, l$, represents the sample average approximations for $\bar{r}_{k}\left(\mathbf{x}^{l}\right)$, and $\boldsymbol{\kappa}_{k}^{l}$ is an approximation for the gradient $\nabla f_{k}\left(\mathbf{x}^{l}\right)$, which is updated recursively as

$$
\boldsymbol{\kappa}_{k}^{l}=\left(1-\beta^{l}\right) \boldsymbol{\kappa}_{k}^{l-1}+\beta^{l} \boldsymbol{\eta}_{k}\left(\mathbf{x}^{l}\right),
$$

with $\boldsymbol{\kappa}_{k}^{-1}=\mathbf{0}$, where $\beta^{l} \in(0,1]$ is the step-size sequence and $\boldsymbol{\eta}_{k}$ is the gradient of the instantaneous rate $r_{k}(\mathbf{x})$ w.r.t. $\mathbf{x}$. Then $\boldsymbol{\eta}_{k}$ can be written as

$$
\boldsymbol{\eta}_{k}(\mathbf{x})=\left[\nabla_{\mathbf{p}}^{T} r_{k}, \nabla_{\mathbf{c}}^{T} r_{k}, \nabla_{\mathbf{v}}^{T} r_{k}, \nabla_{\mathbf{w}}^{T} r_{k}\right]^{T},
$$

where $\nabla_{\mathbf{p}} r_{k}, \nabla_{\mathbf{c}} r_{k}, \nabla_{\mathbf{v}} r_{k}, \nabla_{\mathbf{w}} r_{k}$ are the gradients of $r_{k}$ w.r.t. $\mathbf{p}, \mathbf{c}, \mathbf{v}, \mathbf{w}$, respectively, based on the matrix calculus and the chain rule.

\section{B. Proposed RSSCA Algorithm}

The proposed RSSCA algorithm iteratively minimizes a sequence of surrogate functions. Recalling (9) allows us to express the problem (6) as the following approximated convex one

$$
\min _{\mathbf{x} \in \chi} \sum_{k=1}^{K} p_{k} \quad \text { s.t. } \quad \hat{f}_{k}^{l}(\mathbf{x}) \leq 0, \quad \forall k .
$$

$$
\chi=\left\{\boldsymbol{x}: \boldsymbol{p} \in\left[0, P_{k}^{\max }\right]^{K} ; \sum_{i=1}^{N} c_{i j}=1 ; \sum_{j=1}^{S} c_{i j} \leq 1 ; c_{i j} \in\{0,1\}, \forall i, j\right\}
$$




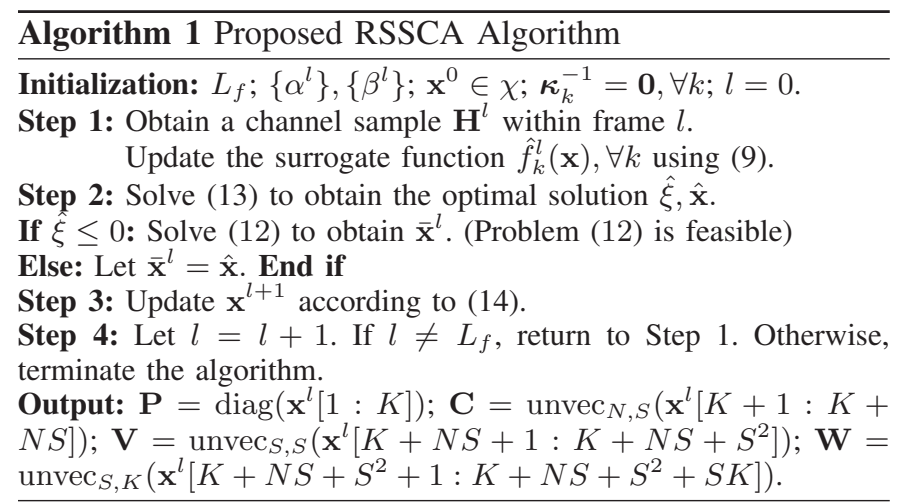

The details of the solution of problem (12) will be postponed to Section III-C. Note that problem (12) may not have a feasible optimal solution. If problem (12) turns out to be infeasible, we have to construct the following feasibility problem.

$$
\min _{\mathbf{x} \in \chi, \xi} \xi \quad \text { s.t. } \quad \hat{f}_{k}^{l}(\mathbf{x}) \leq \xi, \quad \forall k,
$$

which can be efficiently solved by the convex programming toolbox CVX. The solution of problem (13) can be recognized as the projection of problem (12) onto the point, which is the closest to the feasible region of problem (12).

Given the optimal solution $\bar{x}^{l}$ in problem (12) or (13), $\mathrm{x}$ is updated as

$$
\mathbf{x}^{l+1}=\left(1-\alpha^{l}\right) \mathbf{x}^{l}+\alpha^{l} \overline{\mathbf{x}}^{l},
$$

where $\alpha^{l} \in(0,1]$ is the step-size sequence. Then the above iteration is carried out until convergence is reached. We summarize the details of the proposed RSSCA in Algorithm 1,

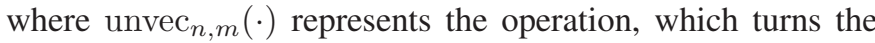
$n m \times 1$ column vector into a matrix of size $n \times m$.

\section{Solutions for Problem (12)}

We provide solutions to the quadratic optimization subproblems in (12) by employing the Lagrange dual method. Once given the Lagrange multipliers, we can obtain a unique closedform solution for the Lagrange function minimization problem. Additionally, the number of dual variables is usually much lower than that of the primal variables $\boldsymbol{x}$ in the quantized mMIMO regime. Accordingly, solving the dual problem is more efficient than directly solving the optimal primal problem.

For the problem at hand, the first step is to introduce the nonnegative Lagrange multipliers $\boldsymbol{\lambda}=\left[\lambda_{1}, \ldots, \lambda_{K}\right]^{T}, \boldsymbol{\mu}=$ $\left[\mu_{1}, \ldots, \mu_{N}\right]^{T}, \varrho=\left[\varrho_{1}, \ldots, \varrho_{K}\right]^{T}, \boldsymbol{\delta}=\left[\delta_{1}, \ldots, \delta_{S}\right]^{T}, \boldsymbol{\Phi}=$ $\left[\phi_{1}, \cdots, \phi_{S}\right]$ associated with the constraints of (12). For clarity, we denote $\Theta=\left[\boldsymbol{\lambda}^{T}, \boldsymbol{\mu}^{T}, \boldsymbol{\varrho}^{T}, \boldsymbol{\delta}^{T}, \operatorname{vec}(\boldsymbol{\Phi})^{T}\right]^{T}$. Then we write the Lagrange function of problem (12) in (15) as displayed at the bottom of this page. Then the dual function for (15) can be expressed as $f_{d}^{l}(\Theta)=\min _{\mathbf{x}} \mathcal{L}^{l}(\mathbf{x}, \Theta)$ and the corresponding dual problem is given by

$$
\max _{\Theta} f_{d}^{l}(\Theta) \text {. }
$$

Note that $f_{d}^{l}(\Theta)$ can be further decomposed into $N$ independent subproblems as $\min _{x_{t} \in \chi_{t}} a(\Theta)\left|x_{t}\right|^{2}+\Re\left[\sum_{t=1}^{n} b(\Theta) x_{t}\right]$, which admits the closed-form solution as

$$
\hat{x}_{t}(\Theta)=\mathbb{P}_{\chi_{t}}\left[-b^{*}(\Theta) / 2 a(\Theta)\right],
$$

where $\mathbb{P}_{\chi_{t}}[\cdot]$ is the projection over the convex set $\chi_{t}$. The optimal Lagrange multipliers $\Theta^{\star}$ of the dual problem in (16) can be solved using the standard subgradient-based method such as the ellipsoid method in [13]. Then the optimal primal solution of (12) is given by $\mathbf{x}^{\star}\left(\Theta^{\star}\right)$.

\section{Convergence Analysis of the Proposed RSSCA Algorithm}

By averaging all the outputs corresponding to the previous feasible updates or objective updates, we can obtain the limiting point $\mathbf{x}$ alternatively. Nevertheless, it is tricky to prove that the limiting point is a stationary point of problem (6). To overcome these challenges, we first introduce the following lemma to guarantee the convergence of the recursive approximation $\hat{r}_{k}^{l}$ and the surrogate function $\hat{f}_{k}^{l}(\boldsymbol{x})$.

Lemma 1: If the step-size sequence $\left\{\alpha^{l}\right\}$ and $\left\{\beta^{l}\right\}$ satisfy the following three conditions

1) $\alpha^{l} \rightarrow 0, \sum_{l} \alpha^{l}=\infty, \sum_{l}\left(\alpha^{l}\right)^{2}<\infty$,

2) $\beta^{l} \rightarrow 0, \sum_{l} \beta^{l}=\infty, \sum_{l}\left(\beta^{l}\right)^{2}<\infty$,

3) $\lim _{l \rightarrow \infty} \alpha^{l} / \beta^{l}=0$,

we almost surely have

$$
\begin{array}{r}
\lim _{l \rightarrow \infty}\left|\hat{r}_{k}^{l}\left(\mathbf{x}^{l}\right)-\bar{r}_{k}\left(\mathbf{x}^{l}\right)\right|=0, \\
\lim _{l \rightarrow \infty}\left|\hat{f}_{k}^{l}\left(\mathbf{x}^{l}\right)-f_{k}\left(\mathbf{x}^{l}\right)\right|=0, \\
\lim _{l \rightarrow \infty}\left\|\nabla \hat{f}_{k}^{l}\left(\mathbf{x}^{l}\right)-\nabla f_{k}\left(\mathbf{x}^{l}\right)\right\|=0 .
\end{array}
$$

Moreover, consider a subsequence $\left\{\mathbf{x}^{l_{j}}\right\}_{j=1}^{\infty}$ converging to a limiting point $\mathrm{x}^{\star}$, and define

$\tilde{f}_{k}(\mathbf{x}) \triangleq \gamma_{k}-\bar{r}_{k}\left(\mathbf{x}^{\star}\right)+\Re\left[\nabla^{H} f_{k}\left(\mathbf{x}^{\star}\right)\left(\mathbf{x}-\mathbf{x}^{\star}\right)\right]+\tau_{k}\left\|\mathbf{x}-\mathbf{x}^{\star}\right\|^{2}$.

Then we almost surely have $\lim _{j \rightarrow \infty} \hat{f}_{k}^{l_{j}}(\mathbf{x})=\tilde{f}_{k}(\mathbf{x}), \forall \mathbf{x} \in \chi$.

Lemma 1 can be proven following an approach similar to that in [15]. Thus, the details are omitted due to the limited space. According to (18)-(20) in Lemma 1, we may infer that the objective value and the gradient of the surrogate function $\hat{f}_{k}^{l}$ as well as the recursive approximation $\hat{r}_{k}^{l}$ are unbiased estimates of $f_{k}\left(\mathbf{x}^{l}\right)$ and $\bar{r}_{k}^{l}\left(\mathbf{x}^{l}\right)$, respectively. In the sequel, we briefly discuss the motivation for some conditions on $\left\{\alpha^{l}, \beta^{l}\right\}$. Firstly, condition 1) and 2) state that both the step-size $\alpha^{l}$ and $\beta^{l}$ satisfy the diminishing rule, namely, do not decay to zero sharply. Without loss of generality, the diminishing step-size rule has also been proposed in many other stochastic optimization solutions [15]. Secondly, condition 3) clarifies

$$
\begin{aligned}
\mathcal{L}^{l}(\mathbf{x}, \Theta) & =\sum_{k=1}^{K} p_{k}+\sum_{k=1}^{K} \lambda_{k} \hat{f}_{k}^{l}+\sum_{i=1}^{N} \mu_{i}\left(\sum_{j=1}^{S} c_{i j}-1\right)+\sum_{k=1}^{K} \varrho_{k}\left(p_{k}-P_{k}^{\max }\right)+\sum_{j=1}^{S} \delta_{j}\left(\sum_{i=1}^{N} c_{i j}-1\right)+\sum_{i=1}^{N} \sum_{j=1}^{S} \phi_{i j}\left(c_{i j}-1\right) \\
& =\sum_{t=1}^{n} a(\Theta)\left|x_{t}\right|^{2}+\Re\left[\sum_{t=1}^{n} b(\Theta) x_{t}\right]+c(\Theta)
\end{aligned}
$$


TABLE I

COMPUTATIONAL COMPLEXITY ORDERS FOR DIFFERENT SCHEMES

\begin{tabular}{cc}
\hline Schemes & Computational complexity order per iteration \\
\hline SHC scheme & $\mathcal{O}\left(\left(N^{3} S^{3}+N^{2} S^{4}+N S^{4}+S^{2} K^{2}\right) \log (1 / \epsilon)+S^{2} K+S\left(N K+K^{2}\right)\right)$ \\
MM scheme & $\mathcal{O}\left(\left(4 S^{2} K^{2}+4 S K^{3}\right) \log (1 / \epsilon)+S^{2} K+S K^{2}\right)$ \\
ZF scheme & $\mathcal{O}\left(\left(N^{3} S^{3}+N^{2} S^{3} K+N S^{3}+S^{2} K^{2}\right) \log (1 / \epsilon)+S\left(K^{2}+N K\right)+K^{3}\right)$ \\
MRC scheme & $\mathcal{O}\left(\left(N^{3} S^{3}+N^{2} S^{3} K+N S^{3}+S^{2} K^{2}\right) \log (1 / \epsilon)+S\left(K^{2}+M K+N K\right)\right)$ \\
\hline
\end{tabular}

that the diminishing speed of $\alpha^{l}$ is faster than that of $\beta^{l}$. With Lemma 1, we can have the following theorem.

Theorem 3.1: Suppose the above assumptions are satisfied. For any sequence $\left\{\mathbf{x}^{t_{j}}\right\}_{j=1}^{\infty}$ converging to a limiting point $\mathbf{x}^{\star}$, problem (6) almost surely converges to stationary point $\mathrm{x}^{\star}$ if the Slater condition is satisfied.

E. Computational Complexity

In this subsection, we compare the computational complexity of the proposed RSSCA algorithm to that of the following baseline schemes.

- Baseline 1-magnitude maximization (MM) scheme: This is obtained by selecting a set of codewords according to the maximum SNR criterion [16].

- Baseline 2-zero-forcing (ZF) scheme: This is obtained by fixing the $\mathrm{ZF}$ digital combiner [1].

- Baseline 3-maximum ratio combining (MRC) scheme: This is obtained by fixing the MRC digital combiner [5]. For simplicity, we assume that $M \gg N \geq S \geq K$. We first analyze the computational complexity of the proposed RSSCA algorithm. Then, the computational complexity order of the other schemes can be obtained similarly. The complexity of the proposed RSSCA algorithm is dominated by the calculation of the gradient $\nabla f_{k}\left(\mathbf{x}^{l}\right)$ and the quadratic optimization subproblems in (12) and (13), which is elaborated on below. Calculating $\nabla f_{k}\left(\mathbf{x}^{l}\right)$ requires $\mathcal{O}\left(K\left(K+N S+S^{2}+K S\right)\right)$ floating point operations (FPOs). For the given Lagrange multipliers, calculating the closed-form primal solution in (17) needs $\mathcal{O}\left(K+N S+S^{2}+K S\right)$ FPOs. Using the ellipsoid method, the number of iterations required for achieving a given convergence accuracy $\epsilon$ for the dual problem $f_{d}^{l}(\Theta)=\min _{\mathbf{x}} \mathcal{L}^{l}(\mathbf{x}, \Theta)$ is $\mathcal{O}\left((2 K+N+S+N S)^{2} \log (1 / \epsilon)\right)$ [13]. Hence, the periteration calculation needs $\mathcal{O}\left(\left(K+N S+S^{2}+K S\right)(2 K+\right.$ $\left.N+S+N S)^{2} \log (1 / \epsilon)+K\left(K+N S+S^{2}+K S\right)\right)$ FPOs.

In Table I, we summarize the computational complexity orders of the different schemes. For the $\mathrm{ZF}$ scheme this is $\mathcal{O}\left(\left(N^{3} S^{3}+N^{2} S^{3} K+N S^{3}+S^{2} K^{2}\right) \log (1 / \epsilon)+S\left(K^{2}+N K\right)+\right.$ $\left.K^{3}\right)$ because in addition to the calculation of the gradient and the quadratic optimization subproblems it requires the calculation of inversion, when adopting the $\mathrm{ZF}$ digital combiner. Furthermore, the complexity order of the MRC scheme can be analyzed in a similar way. Although the MM scheme imposes a slightly lower computational complexity, its performance is in general much worse than that of our proposed SHC scheme. Consequently, our proposed SHC scheme strikes a better performance vs. complexity trade-off.

\section{F. Implementational Considerations}

Next, we investigate the pilot overhead and quantify the advantages over the ICSI-based scheme as follows. Specifically, the overall pilot overheads of the SHC and ICSI- based schemes are $O\left(K M L_{c}\right)$ and $O\left(K M L_{f} L_{s}\right)$, respectively, where $L_{c}$ is the number of frames required by the proposed algorithm. Hence, the SHC scheme advocated reduces the pilot overhead by a factor of $\frac{L_{c}}{L_{f} L_{s}}$. Then, we compare the robustness of different schemes in terms of the feasible probability of the average rate constraint (6b) when $M=64, S=12$, and $K=12$. By averaging over 500 independent channel realizations, the feasible probability of the proposed SHC and the ICSI-based schemes is $90.05 \%$ and $54.53 \%$, respectively. The reason is that the performance of the ICSI-based scheme is crucially dependent on the CSI estimated at each time slot, and having ICSI errors caused by the delay are inevitable in practice due to the limited training resources. However, the acquisition of CSI in the SHC scheme is performed at each frame with higher accuracy. The delayed CSI is generated based on the autoregressive model of [14]. In short, the proposed $\mathrm{SCH}$ scheme is more robust to the CSI signaling delay than the ICSI-based scheme due to the sophisticated stochastic design and owing to the reduction of signalling bits.

In this section, we numerically evaluate the performance of our proposed scheme and glean useful insights. We consider the scenario of $M=64$ receive antennas, $S=12 \mathrm{RF}$ chains, and $N=16$ DFT codewords. The maximum transmit power at each user is $P_{k}^{\max }=10 \mathrm{dBm}$. We set the same average target rate for all users, namely $\gamma_{k}=1 \mathrm{bps} / \mathrm{Hz}$. The BS's coverage has a radius of $200 \mathrm{~m}$ and $K=12$ users are randomly located. Additionally, the channel between user $k$ and the $\mathrm{BS}$ is generated according to the extended Saleh-Valenzuela geometric model using a half-wavelength ULA [15]. We use the large-scale pathloss model $30.6+36.7 \log _{10}\left(d_{k}\right)$ [17] to characterize the average channel gain, where $d_{k}$ is the individual distance between user $k$ and the $\mathrm{BS}$ in meters. The initial parameters are set as $\beta=1 /(1+l)^{2 / 3}$ and $\alpha=5 /(5+l)$, respectively. Four schemes are included as benchmarkers: 1) MM scheme; $\mathbf{C}$ concentrating on choosing the beams with maximum magnitude [16]; 2) random scheme, which is obtained by randomly associating each RF chain with the specific beam; 3) ZF scheme; 4) MRC scheme.

In Fig. 2, we show the objective function (average transmit power) and the maximum constraint function (target average rate) versus the number of iterations for $q=3$ quantization bits, respectively. We can see that the proposed RSSCA algorithm converges within a few iterations, while all the target average rates are strictly satisfied with high accuracy.

In Fig. 3 (a), we plot the average transmit power versus the numbers of users $K$ for $q=4$ quantization bits. It can be seen that the performance of the proposed SHC scheme is 

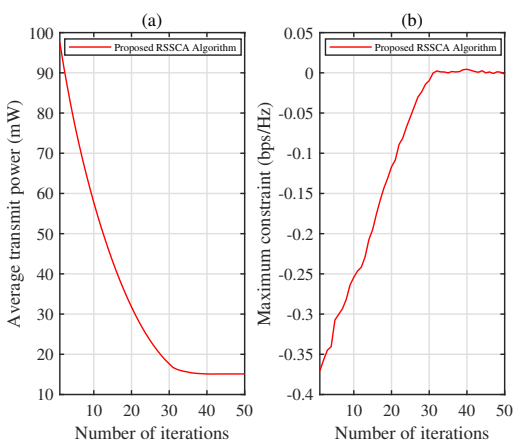

Fig. 2. Convergence behavior of the proposed RSSCA algorithm with $M=$ 64 antennas, $K=12$ users, $S=12 \mathrm{RF}$ chains, $N=16$ DFT codewords and $q=3$ quantization bits: (a) average transmit power; (b) maximum constraint.

superior to that of all the other competing schemes. When the number of users increases, the performance gap between the proposed SHC scheme and these other schemes is widened, which also demonstrates the necessity of joint power control and hybrid combiner design for the quantized mMIMO system. Thus, it appears that for the quantized mMIMO relying on limited resources, our proposed scheme is particular appealing from an optimum resource allocation perspective. Fig. 3 (b) depicts the average transmit power versus the number of BS antennas $M$ with 4-bits ADCs. We observe that the proposed SHC scheme significantly outperforms all the other competing schemes, as expected. It is interesting to note that the benefit of increasing the number of BS antennas is more pronounced for our proposed scheme. The reason is that our proposed scheme can exploit the difference in channel quality among links for mitigating the multiuser interference, thereby supporting more favorable uplink transmission in a cost-effective manner. Fig. 3 (c) plots the system's average transmit power versus the number of quantization bits $q$. It shows that the average transmit power is monotonically decreasing as the number of quantization bits increases. Furthermore, the proposed SHC scheme outperforms all the other schemes in all quantization scenarios, especially for more than 3 quantization bits.

A novel SHC scheme was conceived for the uplink of quantized mMIMO systems for minimizing the transmit power consumption under an average rate constraint. We proposed a RSSCA algorithm to find stationary solutions of the nonconvex stochastic optimization problem. We demonstrated that the proposed SHC scheme outperforms the benchmarkers.

\section{REFERENCES}

[1] E. Björnson et al., "Massive MIMO networks: Spectral, energy, and hardware efficiency," Foundations and Trends in Signal Processing, vol. 11 , no. 3-4, pp. 154-655, Nov. 2017.

[2] S. Park et al., "Exploiting spatial channel covariance for hybrid precoding in massive MIMO Systems." IEEE Trans. Signal Process., vol. 65, no. 9, pp. 3818-3832, Jul. 2017.

[3] V. Venkateswaran et al., "Analog beamforming in MIMO communications with phase shift networks and online channel estimation," IEEE Trans. Signal Process., vol. 58, no. 8, pp. 4131-4143, Aug. 2010.

[4] K. Roth et al., "A comparison of hybrid beamforming and digital beamforming with low-resolution ADCs for multiple users and imperfect CSI," IEEE J. Sel. Topics Signal Process., vol. 12, no. 3, pp. 484-498, Jun. 2018.

[5] J. Choi et al., "Two-stage analog combining in hybrid beamforming systems with low-resolution ADCs," IEEE Trans. Signal Process., vol. 67, no. 9, pp. 2410-2425, May 2019.

[6] H. Sheng et al., "Energy efficiency optimization for beamspace massive MIMO systems with low-resolution ADCs," in 2020 IEEE Wireless Communi. Netw. Conf. (WCNC), Seoul, Korea, Jun. 2020, pp. 1-7.
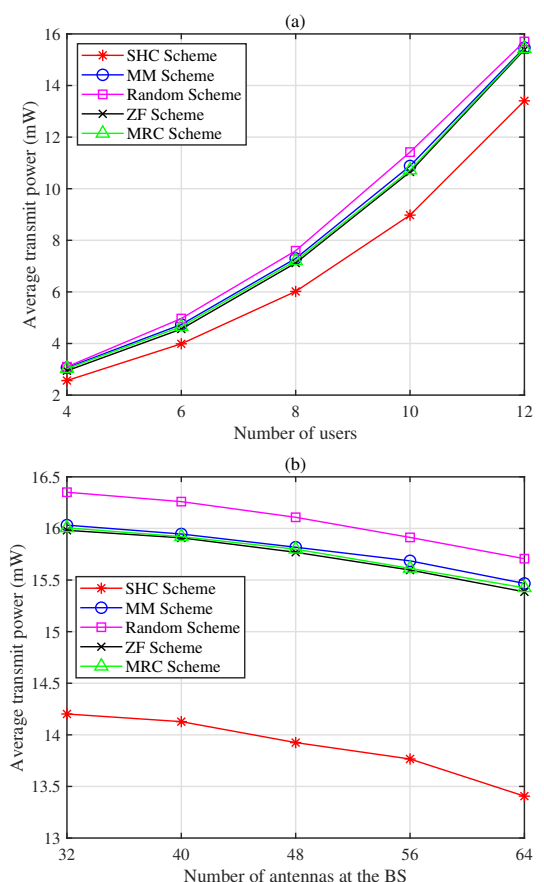

(c)

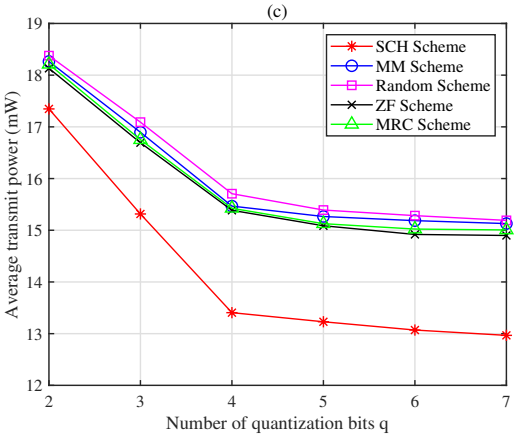

Fig. 3. (a) Average transmit power versus the number of users with $M=64$ antennas and 4-bits ADCs; (b) average transmit power versus the number of antennas at the BS with $K=12$ users and 4-bits ADCs; (c) average transmit power versus the number of quantization bits $q$ with $M=64$ antennas and $K=12$ users.

[7] M. B. Khalilsarai et al., "FDD massive MIMO via UL/DL channel covariance extrapolation and active channel sparsification," IEEE Trans. Wireless Commun., vol. 18, no. 1, pp. 121-135, Jan. 2019.

[8] Y. Chen et al., "Fundamental trade-offs on green wireless networks," IEEE Commun. Mag., vol. 49, no. 6, pp. 30-37, Jun. 2011.

[9] T. Liu et al., "Energy efficiency of massive MIMO systems with lowresolution ADCs amd successive interference cancellation," IEEE Trans. Wireless Commun., vol. 18, no. 8, pp. 3987-4002, Aug. 2019.

[10] T. L. Marzetta et al., Fundamentals of Massive MIMO. Cambridge, U.K.: Cambridge Univ. Press, 2016.

[11] S. Wagner et al., "Large system analysis of linear precoding in correlated MISO broadcast channels under limited feedback," IEEE Trans. Info. Theory, vol. 58, no. 7, pp. 4509-4537, Jul. 2012.

[12] L. Fan et al.,"Uplink achievable rate for massive MIMO systems with low-resolution ADC," IEEE Commun. Lett., vol. 19, no. 12, pp. 2186$2189,2015$.

[13] S. Boyd et al., Convex optimization. Cambridge, U.K.: Cambridge Univ. Press, 2004.

[14] A. Liu et al., "Two-timescale hybrid compression and forward for massive MIMO aided C-RAN," IEEE Trans. Signal Process., vol. 67, no. 9, pp. 2484-2498, Mar. 2019.

[15] A. Liu et al., "Stochastic successive convex optimization for twotimescale hybrid precoding in massive MIMO," IEEE J. Sel. Topics Signal Process., vol. 12, no. 3, pp. 432-444, Jun. 2018.

[16] A. Sayeed et al., "Beamspace MIMO for high-dimensional multiuser communication at millimeter-wave frequencies," in Proc. IEEE Global Commun. Conf. (GLOBECOM), Dec. 2013, pp. 3679-3684.

[17] Technical Specification Group Radio Access Network: Further Advancements for E-UTRA Physical Layer Aspects, 3GPP TR 36.814. [Online]. Available: http://www.3gpp.org 